\title{
A Computationally Efficient Abstraction of Long-term Potentiation
}

\author{
Lokendra Shastri \\ International Computer Science Institute \\ 1947 Center Street, Suite 600 \\ Berkeley, CA 94704, USA \\ shastri@icsi.berkeley.edu
}

\begin{abstract}
Key words: Long-term potentiation; hippocampus; binding detection; dentate gyrus; episodic memory
\end{abstract}

\begin{abstract}
A computational abstraction of long-term potentiation (LTP) is proposed. The abstraction captures key temporal and cooperative properties of LTP, and also lends itself to rapid computation. The abstraction is used to simulate the recruitment of binding-detector cells in the dentate gyrus (DG). The simulation results are used to validate a quantitative analysis of binding-detector cell recruitment in DG. The analysis shows that (i) a large number of bindingdetector cells would be recruited in response to entorhinal cortex activity and (ii) cells recruited for distinct bindings would exhibit very low cross-talk. These results help in understanding the neural basis of episodic memory.
\end{abstract}

\section{Introduction}

Long-term potentiation (LTP) refers to a long-term increase in synaptic strength resulting from the pairing of presynaptic activity with postsynaptic depolarization $[3,10]$. Recent evidence strongly suggests that LTP plays a direct causal role in learning and memory formation (e.g., $[13,19])$. LTP was first observed in the rabbit hippocampal formation, and has since been observed in synapses along many excitatory pathways in the mammalian brain. The most extensively studied form of LTP involves the unusual receptor $\mathrm{NMDA}^{\mathbf{1}}$ ( $N$-methyl-D-aspartate) which is activated by the excitatory neurotransmitter glutamate, but only if the postsynaptic membrane is sufficiently depolarized. In the absence of adequate depolarization, NMDA receptor-gated channels remain blocked by magnesium ions. Adequate depolarization of the postsynaptic membrane, however, expels the magnesium ions and unblocks the channels enabling calcium ions to flood into the dendritic spine and trigger biochemical changes that result in the induction of LTP.

The two conditions required for the activation of NMDA receptor, namely, presynaptic activity and strong postsynaptic depolarization, together entail that LTP requires the concurrent arrival of activity at several synapses of the postsynaptic cell. This is referred to as the cooperativity property of LTP $[12,8]$. The cooperativity property of LTP makes it an ideal mechanism for transforming a transient expression of a relationship between two or more items (encoded as the coherent activity of the ensembles representing these items) into a persistent expression of this relationship (encoded via long-term changes in the efficacy of synapses that link ensembles representing these items). Several other properties of LTP also make it suitable for memorization - it is induced rapidly, it is long lasting, and since it is synapse specific, it can express highly specific bindings and correlations.

LTP resulting from activity along afferent fibers belonging to a single pathway is referred to as homosynaptic LTP. If the arrival of activity along two pathways, $A$ and $B$, leads to LTP of synapses formed by fibers of $A$, but the arrival of activity along fibers of $A$ alone does not, then the LTP of synapses formed by fibers of $A$ is referred to as associative LTP $[5,8]$. We will, however, distinguish between associative and homosynaptic LTP based on the representational distinctiveness of the afferent sources. Thus we will use the qualifier "associative" for LTP resulting from the arrival of coincident activity along afferent fibers emanating from cells representing distinct items.

Synapses along key excitatory pathways in the hippocampus also undergo long-term depression (LTD) [9]. A synapse receiving no presynaptic activity can undergo heterosynaptic LTD if other synapses of the postsynaptic cell receive strong presynaptic activity, and low frequency stimulation of a synapse can lead to its homosynaptic LTD.

\footnotetext{
${ }^{1}$ Not all forms of LTP are NMDA receptor-dependent. The LTP of synapses formed by mossy-fi bers on CA3 pyramidal cells is a case in point.
} 
A computational abstraction of LTP is proposed that captures key temporal and cooperative properties of LTP, but lends itself to rapid computation. This abstraction is used to simulate the recruitment of binding-detector (or coincidence-detector) cells in the dentate gyrus (DG), and results of these simulations are used to validate a statistical analysis of binding-detector cell recruitment in DG. The validated analysis is applied to a full-scale model of DG.

\section{A Computational Abstraction of Cells and Synapses}

The computational abstraction of LTP and LTD proposed here is an idealization of a complex biophysical processes. This abstraction is guided by two considerations. First, the abstraction should be rich enough to capture key temporal aspects of LTP and LTD. Second, the abstraction should be discrete and minimal so as to facilitate quantitative analyses and efficient computer simulations of large-scale neuronal networks containing thousands of cells and several hundred thousand synapses using off-the-shelf workstations.

As explained below, the abstraction requires only integer valued parameters and integer arithmetic operations. The proposed abstraction is much more detailed than those used in artificial neural networks and connectionist models, but far less detailed than those used in biophysically detailed simulations (e.g., using GENESIS [4] and NEURON [7]).

A cell is abstracted as an integrate-and-fire neuron (e.g., see [11]), and the spatio-temporal integration of activity arriving at a cell is modeled as follows: Let $w_{i}(t)$ refer to the weight of synapse $s_{i}$ at time $t$, and let $a_{i}(t)$ be a measure of presynaptic activity occurring at synapse $s_{i}$ at time $t$. In biophysical terms, $a_{i}(t)$ may correspond to the number of spikes arriving at $s_{i}$ within a unit time interval anchored at $t$. Thus the arrival of a high-frequency spike-burst at $s_{i}$ would correspond to a high value of $a_{i}(t)$. The postsynaptic potential, $\operatorname{psp}_{i}\left(t \mid a_{i}\left(t_{0}\right)\right)$, resulting from the presynaptic activity at $s_{i}$ at time $t_{0}$ is modeled as a piecewise linear function consisting of a rising segment, a flat segment, and a falling segment. That is:

$$
\operatorname{psp}_{i}\left(t \mid a_{i}\left(t_{0}\right)\right)= \begin{cases}m_{r} *\left(t-t_{0}\right) & t_{0} \leq t<\left(t_{0}+\Delta T_{r}\right) \\ m_{r} * \Delta T_{r} & \left(t_{0}+\Delta T_{r}\right) \leq t<\left(t_{0}+\Delta T_{r s}\right) \\ m_{r} * \Delta T_{r}+\left(t-\Delta T_{r s}\right) * m_{f} & \left(t_{0}+\Delta T_{r s}\right) \leq t<\left(t_{0}+\omega_{\text {int }}\right) \\ 0 & \text { otherwise }\end{cases}
$$

where $\Delta T_{r}$ is the duration of the rising segment, $m_{r}$ is the slope of the rising segment and is given by $\left(a_{i}\left(t_{0}\right) *\right.$ $\left.w_{i}\left(t_{0}\right)\right) / \Delta T_{r}, \Delta T_{s}$ is the duration of the flat segment, $\Delta T_{r s}$ equals $\left(\Delta T_{r}+\Delta T_{s}\right), m_{f}$ is the slope of the falling segment, and $\omega_{i n t}$ is the window of temporal integration denoting the maximum amount by which two incident activities may lead/lag and still be summated by the postsynaptic cell. Note that $\omega_{i n t}=\Delta T_{r s}+\left(m_{r} * \Delta T_{r}\right) / m_{f}$.

The postsynaptic potential at time $t$ attributable to $s_{i}, p s p_{i}(t)$, can be obtained by summing the effect of all the activity arriving at $s_{i}$ during the past $\omega_{i n t}$ time units. Thus $p s p_{i}(t)=\sum_{\left(0 \leq \tau<\omega_{i n t}\right)} p s p_{i}\left(t \mid a_{i}(t-\tau)\right)$. Moreover, $p o t(t)$, a cell's potential at time $t$ resulting from the combined effect of presynaptic activity at all its synapses equals: $\sum_{i} p s p_{i}(t)$, where $i$ ranges over all synapses of the cell.

A cell has a firing threshold, thresh $h_{f}(t)$, with a resting value of $\theta_{f}$. A cell fires at time $t$ if $\operatorname{pot}(t) \geq t h r e s h_{f}(t)$, and produces an action potential (spike). This spike arrives at synapses downstream from the cell at time $t+d$, where $d$ is a variable propagation delay. After firing, a cell enters an absolute refractory state for a duration $\omega_{\text {ref }}$.

Cells can have multiple firing modes - each mode being associated with different firing thresholds and output levels. For example, one mode may correspond to a simple spike response consisting of isolated spikes, while another mode may correspond to a high-frequency burst response such as the complex spike burst response generated by hippocampal pyramidal cells. For suitable choices of parameter values, the simple abstraction of a firing mode offers a computationally inexpensive means of modeling distinct response modes of cells.

A synapse can be in any one of following three states: naive, potentiated, or depressed. The state of a synapse signifies its strength (weight). For a given synaptic type, the weights of all synapses in a given state lie within a restricted band, and the weight bands associated with different states are disjoint. The weight bands associated with a synaptic state may differ from one synaptic type to another.

\section{Computational abstraction of LTP and LTD}

The induction of LTP is governed by the following parameters: the potentiation threshold $\theta_{p}$, the weight increment $\Delta w_{l t p}$, the repetition factor $\kappa$, and the maximum inter-activity interval $\tau_{i a i}$. 
Consider a set of synapses $s_{1}, \ldots, s_{n}$ sharing the same postsynaptic cell. Convergent presynaptic activity at $s_{1}, \ldots, s_{n}$ can lead to LTP of naive $s_{i}$ 's and increase their weights by $\Delta w_{l t p}$ if the following conditions hold: (i) $\sum_{1 \leq i \leq n} p s p_{i}(t) \geq \theta_{p}$ (recall that in order to summate, the presynaptic activity must be "synchronous" and arrive within a window of width $\omega_{i n t}$ ); (ii) such synchronous presynaptic activity repeats at least $\kappa$ times, and (iii) the interval between two successive arrivals of presynaptic activity during the above repetition is at most $\tau_{i a i}$.

In the case of homosynaptic LTP, the activity leading to LTP emanates from a cell ensemble representing a single item. In the case of associative LTP such activity emanates from cell ensembles representing more than one item.

Heterosynaptic LTD is modeled similarly using five parameters: $\theta_{p}$, weight decrement $\Delta w_{l t d}, \kappa, \tau_{i a i}$, and the propensity of $L T D \zeta 0 \leq \zeta \leq 0$. When synapses of a cell receive presynaptic activity, neighboring inactive naive synapses of the postsynaptic cell undergo heterosynaptic LTD and their weights decrease by $\Delta w_{\text {ltd }}$. Parameters $\theta_{p}, \kappa$, and $\tau_{i a i}$ refer to presynaptic activity in neighboring synapses. The parameter $\zeta$ specifies the fraction of inactive naive synapses that undergo LTD when the above conditions are met.

The effect of neuromodulators on cell firing and LTP induction is a complex phenomena. In the present proposal, these effects are modeled by positing an additional input (or bias) that modifies the firing thresholds of a cell and the potentiation threshold of a synapse.

\section{Simulating the recruitment of binding-detector cells in the dentate gyrus}

The proposed abstraction of LTP has been used to simulate the recruitment of binding-detector (bind) cells in DG in response to EC activity. The functional significance of bind cells is discussed in brief here; a detailed discussion appears in $[14,15,16]$. We remember our experiences in terms of events and situations. This form of memory is referred to as episodic memory [20], and it is known that the hippocampal formation and neighboring cortical areas play a critical role in the formation of such memories [6]. The episodic memory trace of an event must be capable of encoding role-entity bindings. Consider an event, $E$, described by "John gave Mary a book in the library on Tuesday". This event cannot be encoded by simply forming a conjunctive association between "John", "Mary", "a book", "Library", "Tuesday" and "give." In order to make the necessary distinctions the encoding of an event must specify the bindings between the entities participating in the event and the roles they play in the event. For example, the encoding of $E$ should specify the bindings: $\langle$ giver $=\mathrm{John}\rangle,\langle$ recipient=Mary $\rangle,\langle$ give-object $=\mathrm{a}-\mathrm{Book}\rangle,\langle$ temporallocation $=$ Tuesday $\rangle,\langle$ location $=$ Library $\rangle$.

In view of the above, the recruitment of binding-detector (bind) cells is expected to be a critical step in the memorization of episodic memory. The following describes how bind cells may be recruited in DG as a result of EC activity. A discussion of how an event's complete memory trace is encoded in the hippocampal system appears in [16].

It is assumed that roles and entities are encoded as cell ensembles in subregions ECro and ECee of EC, respectively. The projections from high-level cortical circuits (HLCCs) to ECro and ECee correspond to the well known cortical projections to EC, and the projections from ECro and ECee to DG correspond to the dense perforant path projection from EC to DG [2].

It is assumed that bindings constituting an event are expressed as a transient pattern of rhythmic activity over distributed HLCCs. [1, 17, 18, 21]. These HLCCs project to ECro and ECee and induce transient rhythmic activity in these regions. Fig. 1(a) shows an idealized depiction of the activity induced in ECro and ECee by HLCCs to convey the role-entity bindings $\left(\left\langle r_{1}=f_{1}\right\rangle,\left\langle r_{2}=f_{2}\right\rangle\right)$. Here $r_{1}$ and $r_{2}$ are roles, and $f_{1}$ and $f_{2}$ are entities. It is speculated that the dynamic expression of role-entity bindings may involve $\gamma$ band activity and blocks of repeated activity may correspond to $\theta$ band activity.

\subsection{Recruitment of bind cells for memorizing role-entity bindings}

Model DG contains two kinds of cells: principal cells (these correspond to granule cells) and Type-1 inhibitory interneurons (see Fig. 1(b)). Each principal cell receives afferents from cells in ECro and ECee, and makes synaptic contacts on a number of interneurons. The interneurons in turn make contacts with principal cells, thereby forming inhibitory circuits within DG. These inhibitory circuits serve as soft-winner-take-all networks (soft-WTA) and allow synapses of only a limited number of cells to undergo LTP (see [16] for details).

The potentiation threshold, $\theta_{p}$, of principal cells is such that LTP of a synapse occurs only if multiple synapses of a postsynaptic cell receive coincident presynaptic activity. Furthermore, the response threshold, $\theta_{f}$, of principal cells is such that a cell does not fire unless it receives impulses at multiple potentiated synapses. A set of parameter 

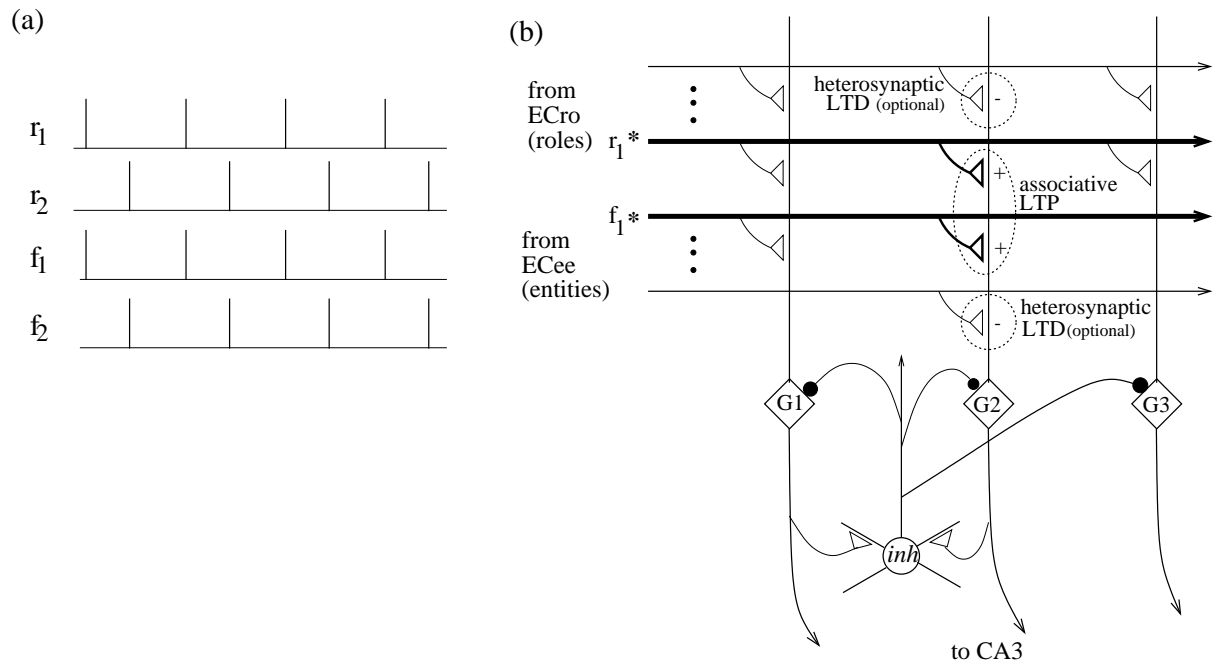

Figure 1: (a) The activity in ECro and ECee encoding the bindings $\left\langle r_{1}=f_{1}\right\rangle$ and $\left\langle r_{2}=f_{2}\right\rangle$. Here $r_{1}$ and $r_{2}$ are roles, and $f_{1}$ and $f_{2}$ are entities. Cells in the $r_{1}$ and $f_{1}$ ensembles fire in synchrony and so do cells in the $r_{2}$ and $f_{2}$ ensembles. Each spike signifies the synchronous firing of a cell ensemble. (b) Recruitment of bind cells in the dentate gyrus (DG) in response to activity in entorhinal cortex. G1-G3 are principal cells and inh is a Type-1 interneuron. Afferents $r_{1} *$ and $f_{1} *$ are from cells in role $r_{1}$ and entity $f_{1}$ ensembles, respectively. Synapses of G1 and G2 receive sufficient synchronous activity along afferents from $r_{1}$ and $f_{1}$ cells, and hence, are candidate binding-detector cells for the binding $\left\langle r_{1}=f_{1}\right\rangle$. While inhibition from $i n h$ prevents LTP of G1's synapses, G2's synapses undergo LTP, and hence, G2 gets recruited as a binding-detector cell for $\left\langle r_{1}=f_{1}\right\rangle$. Filled blobs denote inhibitory synapses, and the size of the blob conveys synaptic strength. Synapses undergoing LTP are marked with a ' + '.

values used in the simulations of DG are as follows: $\theta_{p}=300, \omega_{\text {int }}=5 ; \omega_{\text {ref }}=4 ; \kappa=3 ; w$ (naive) $=100$; $\Delta w_{l t p}=100 ; \theta_{f}=600 ; \tau_{i a i}>\omega_{i n t} * n$, where $n=$ number of bindings. LTD does not play a role in the recruitment of binding-detector cells described here.

The transient encoding of the bindings $\left(\left\langle r_{1}=f_{1}\right\rangle,\left\langle r_{2}=f_{2}\right\rangle\right)$ shown in Fig. 1(a) leads to the following events in DG (refer to Fig. 1(b)). As a result of the synchronous firing of $r_{1}$ and $f_{1}$ cells in ECro and ECee, respectively, certain DG principal cells receive sufficient synchronous inputs, and their active synapses undergo associative LTP. We will refer to such cells as $\operatorname{bind}\left(\left\langle r_{1}=f_{1}\right\rangle\right)$ cells.

Since $\theta_{f}$ of principal cells is such that a cell does not fire unless it receives impulses at multiple potentiated synapses, impulses arriving at numerous naive synapses do not lead to the firing of a $\operatorname{bind}\left(\left\langle r_{1}=f_{1}\right\rangle\right)$ cell. Only the coincident arrival of impulses at many potentiated synapses (for example, from $r_{1}$ and $f_{1}$ cells) satisfies $\theta_{f}$ and causes such a cell to fire. Thus most $\operatorname{bind}\left(\left\langle r_{1}=f_{1}\right\rangle\right)$ cells fire when $r_{1}$ cells in ECro fire in synchrony with $f_{1}$ cells in ECee, and hence, behave as binding-detector cells for the role-entity binding $\left\langle r_{1}=f_{1}\right\rangle$. Similar LTP events lead to the recruitment of $\operatorname{bind}\left(\left\langle r_{2}=f_{2}\right\rangle\right)$ cells. Note that bind cells essentially behave as coincidence detectors.

\subsection{Comparison of simulation results and quantitative analysis}

The results obtained by simulating the recruitment of bind cells in DG are shown in Fig. 2(a) alongside the results obtained by a quantitative analysis of the recruitment process using the same parameter values as those used in the simulations. The number of principal cells in the simulated DG, ECro, and ECee are 5000, 250, and 250, respectively. The size of the projective field from EC to DG is 150 (i.e., there are 75,000 links from EC to DG).

\subsection{Quantitative Results applied to a full-scale model of DG}

The close match between the simulation results and the quantitative analysis validates the accuracy of the quantitative analysis. The validated quantitative analysis is applied to a full-scale model of DG. The number of principal cells in DG, ECro, and ECee are assumed to be 0.75 million, 0.75 million, and 15 million, respectively, and the size of EC to DG projective field is assumed to be 17,000. These values are based on [2, 22, 23]. Furthermore, the number of cells 

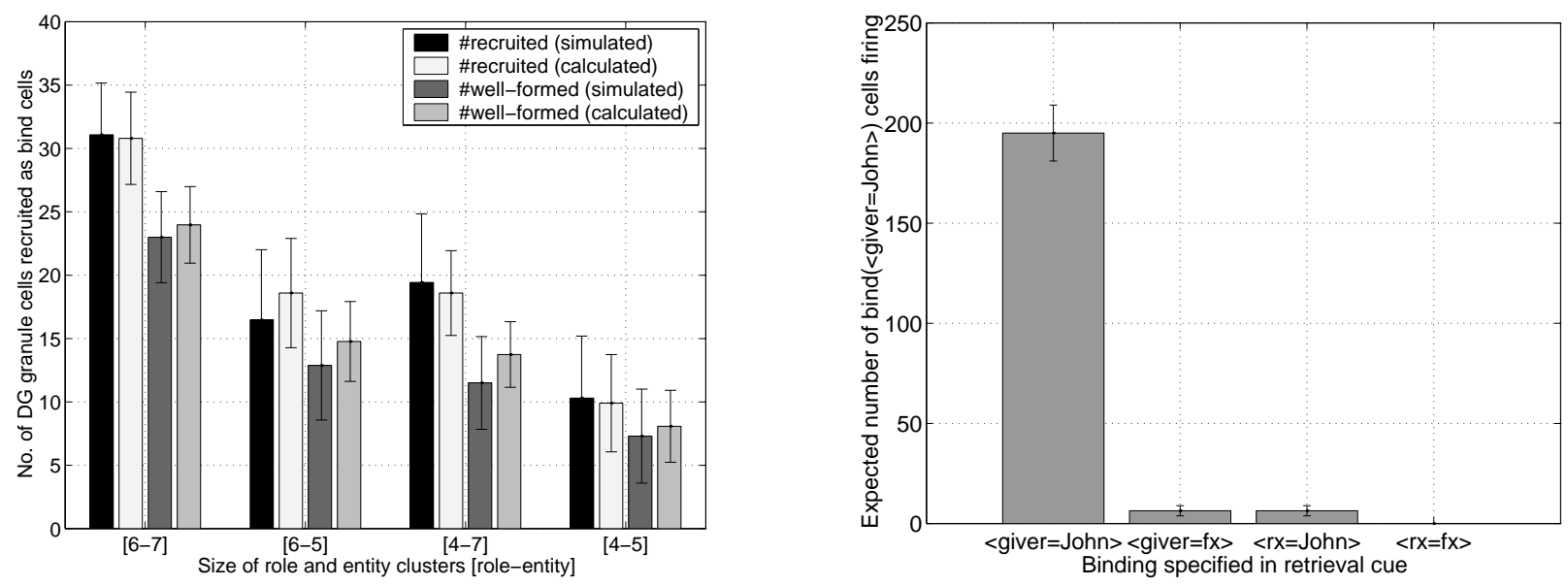

Figure 2: (a) Results of simulating the recruitment of bind cells in DG, and their agreement with results obtained by a quantitative analysis of the model. The total number of DG cells recruited as bind cells and the number of well-formed bind cells recruited are shown. Results are reported for different sizes of role and entity ensembles. For example, the first set of data pertains to the recruitment of bind cells where the role and entity ensembles contain six and seven cells, respectively. Simulation results indicate average values and results of quantitative analysis indicate expected values. For example, the leftmost bar depicts the average number of bind cells recruited (in simulations) for a binding where the role ensemble contains six cells and the entity ensemble contains seven cells. The average depicted by the leftmost bar is based on 12 data points. The number of data points used for other averages range from 12 to 35 . The error-bars denote standard deviations. (b) Response of bind $(\langle$ giver $=$ John $\rangle)$ cells to different retrieval cues. Here "fx" and "rx" refer to entities and roles other than John and giver, respectively.

in each role and entity ensemble is assumed to be 600. Other parameter values used in the analysis are as follows: $\theta_{p}=850, \omega_{i n t}=5 ; \omega_{\text {ref }}=4 ; \kappa=3 ; w$ (naive) $=100 ; \Delta w=100 ; \theta_{f}=1700$. As before, the choice of $\tau_{i a i}$ is governed by $\omega_{\text {int }}$ and the number of role-entity bindings in an event.

The quantitative analysis shows that the probability of not finding any cells in DG to serve as bind cells for a given binding is less than $10^{-18}$ (essentially zero). Furthermore, the expected number of DG cells recruited as bind cells for each binding is 195 (a large number of bind cells are recruited for each binding). The response of bind cells for the binding $\langle$ giver $=J o h n\rangle$ is shown in Fig. 2(b). Note that these cells respond robustly to a matching binding, produce an extremely weak response to erroneous, but partially related bindings, and produce essentially no response to unrelated bindings.

\section{Conclusion}

A computationally efficient abstraction of LTP is proposed. The abstraction is used to simulate the recruitment of binding detector cells in a 5000 cell model of DG. The simulation results are, in turn, used to validate a quantitative analysis of the recruitment process in DG. This quantitative analysis is applied to a full-scale model of DG and it is shown that a sufficient number of bind cells would be recruited in DG in response to EC activation and that bind cells recruited for distinct bindings would exhibit a remarkably low level of cross-talk. These results contribute to an understanding of the neural basis of episodic memory.

\section{References}

[1] V. Ajjanagadde and L. Shastri, Rules and Variables in Neural Nets, Neural Comput. 3 (1991) 121-134.

[2] D.G. Amaral, N. Ishizuka, B. Claiborne, Neurons, numbers and the hippocampal network, in: J. Storm-Mathisen, J. Zimmer, O.P. Ottersen, eds., Progress in Brain Research: Understanding the brain through the hippocampus (Elsevier Science, Amsterdam, 1994) 1-11. 
[3] T.V.P. Bliss and T. Lomo, Long-lasting potentiation of synaptic transmission in the dentate area of the anaesthetized rabbit following stimulation of the perforant path, J. Physiology 232 (1973) 331-356.

[4] J.M. Bower and D. Beeman, The Book of GENESIS: Exploring Realistic Neural Models with the GEneral NEural SImulation System (TELOS/Springer-Verlag, Berlin, 1998).

[5] T.H. Brown, E.W. Kairiss and C.L. Keenan, Hebbian Synapses: Biophysical Mechanisms and Algorithms, Annu. Rev. Neurosci., 13 (1990) 475-511.

[6] N.J. Cohen, and H. Eichenbaum, Memory, Amnesia, and the Hippocampal System (MIT Press, Cambridge, MA, 1993).

[7] M. Hines and J.W. Moore A NEURON simulation program, Abstract for 1995 Soc. Neuroscience Meeting.

[8] W.B. Levy and O. Steward, Synapses as associative memory elements in the hippocampal formation, Brain Res. 175 (1979) 233-245.

[9] D.J. Linden, Long-term synaptic depression in the mamallian brain, Neuron 12 (1994) 457-472.

[10] R.C. Malenka and R.A. Nicoll, Long-term Potentiation - A Decade of Progress? Nature 285 (1999) 1870-1874.

[11] W. Maass and B. Ruf, On Computation with Pulses, Inform. Comput. 148 (1999) 202-218.

[12] B.L. McNaughton, R.M. Douglas and G.V. Goddard, Synaptic enhancement in fascia dentata: Cooperativity among coactive afferents, Brain Res. 157: 277-293.

[13] M.-S. Rioult-Pedotti, D. Friedman, J.P. Donoghue, Learning-induced LTP in Neocortex, Science 290 (2000) 533-536.

[14] L. Shastri, Recruitment of binding and binding-error detector circuits via long-term potentiation, Neurocomputing 26-27 (1999) 865-874.

[15] L. Shastri, A computational model of episodic memory formation in the hippocampal system, Neurocomputing 38-40 (2001) 889-897.

[16] L. Shastri, Episodic memory trace formation in the hippocampal system: a model of cortico-hippocampal interactions. Technical Report 01-004, 2001. International Computer Science Institute, Berkeley, CA. Available at http://www.icsi.berkeley.edu/ shastri/fetex/tr-01-004.pdf.

[17] L. Shastri and V. Ajjanagadde, From simple associations to systematic reasoning. connectionist representation of rules, variables, and dynamic bindings using temporal synchrony, Behav. Brain Sci. 16 (1993) 417-494.

[18] W. Singer and C.M. Gray, Visual feature integration and the temporal correlation hypothesis. Annu. Rev. Neurosci. 18 (1995) 555-586.

[19] Y. Tang, E. Shimizu, G.R. Dube, C. Rampon, G.A. Kerchner, M. Zhuo G. Liu and J.Z. Tsien, Genetic enhancement of learning and memory in mice, Nature 401 (1999) 63 - 69.

[20] E. Tulving, Elements of Episodic Memory (Clarendon Press, Oxford, 1983).

[21] C. von der Malsburg. Am I thinking assemblies? in: G. Palm and A. Aertsen, eds., Brain Theory (SpringerVerlag, Berlin, 1986).

[22] M.J. West, Stereological studies of the hippocampus: a comparison of the hippocampal subdivisions of diverse species including hedgehogs, laboratory rodents, wild mice and men, in: J. Storm-Mathisen, J. Zimmer, and O.P. Ottersen, eds., Progress in Brain Research: Understanding the brain through the hippocampus (Elsevier Science, Amsterdam, 1990) 13-36.

[23] M.J. West and L. Slomianka, Total numbers of neurons in the layers of the human entorhinal cortex, Hippocam-

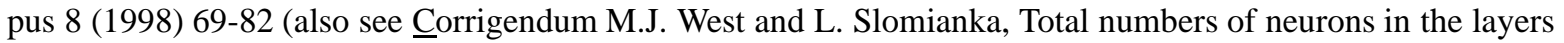
of the human entorhinal cortex. Hippocampus 8 (1998) 69-82. Hippocampus 8 (1998) 426.

Acknowledgments: This work was supported by the NSF grants SBR-9720398 and ECS-9970890. 